\title{
Nanoporous Hybrid Electrolytes
}

\author{
Jennifer L. Nugent, ${ }^{\text {a }}$ Surya S. Moganty, ${ }^{a}$ Dennis A. Yanga, ${ }^{a}$ and Lynden A. Archer ${ }^{* a}$ \\ Received (in XXX, XXX) Xth XXXXXXXXX 200X, Accepted Xth XXXXXXXXX 200X \\ First published on the web Xth XXXXXXXXX 200X \\ DOI: $10.1039 / b 000000 x$
}

Oligomer-suspended $\mathrm{SiO}_{2}$-polyethylene glycol nanoparticles are studied as porous media electrolytes. At $\mathrm{SiO}_{2}$ volume fractions, $\phi$, bracketing a critical value $\phi_{y} \approx 0.29$, the suspensions jam and their mechanical modulus increase by more than seven orders. For $\phi>\phi_{y}$, the mean pore diameter is close to the anion size, yet the ionic conductivity remains surprisingly high and can be understood, at all $\phi$, using a simple effective medium model proposed by Maxwell. $\mathrm{SiO}_{2}$-polyethylene glycol hybrid electrolytes are also reported to manifest attractive electrochemical stability windows (0.3-6.3V) and to reach a steady-state interfacial impedance when in contact with metallic lithium.

\section{Introduction}

Lithium ions are the active charge carrying species in the most energy dense secondary batteries of today, those used in electronics and hybrid electric transportation. Currently commercialized lithiated anode materials such as $\mathrm{LiC}_{6}$ and $\mathrm{Li}_{4} \mathrm{Ti}_{5} \mathrm{O}_{12}$ have relatively low theoretical energy capacities (360 $\mathrm{mAh} / \mathrm{g}$ and $175 \mathrm{mAh} / \mathrm{g}$, respectively). Advanced secondary battery systems employing electrodes such as $\mathrm{LiCoPO}_{4},{ }^{1}$ lithium, ${ }^{2-5}$ or sulfur ${ }^{6}$ require electrolytes with specific properties such as wide electrochemical stability windows, high mechanical strength, and/or inertness or non-solvency towards the electrode materials and their intercalation products. Next-generation lithium ion batteries should also employ electrolytes that are nonflammable, non-volatile, non-leakable, and non-toxic, making them safer both in use and after disposal. In pursuit of such materials, several classes of electrolytes have been studied as replacements for conventional liquid electrolytes: polymers, ${ }^{7-11}$ polymer composites, ${ }^{12-15}$ hybrids, ${ }^{16-18}$ gels, ${ }^{19,20}$ ionic liquids, ${ }^{21}$ and ceramics. ${ }^{22}$

In many cases, mechanical integrity of the electrolyte comes at a cost: namely, a large loss in ionic conductivity, which places undesirable limits on the charge/discharge rate of the cell. Liquid and particulate plasticizers have been used with some success in circumventing this constraint in composite and gel polymer electrolytes. ${ }^{4,15,19,23}$ With a mechanically strong framework in place such as a polymer or ceramic, the liquid plasticizer serves as a freely diffusing ionic conduction medium that provides ionic conductivities near that of a pure liquid electrolyte. If a liquid plasticizer with good thermal and electrochemical properties is utilized, safety concerns are reduced. In the case of particulate plasticizers, of either nano- or micron- scale, the particles have been shown to decrease crystallization of the surrounding matrix, thereby enhancing segmental motion of the host polymer and increasing conduction. While nanoparticles have been shown most successful in this area, in typical polymer composites particle aggregation occurs; this reduces the effectiveness of the individual particles in inhibiting crystallization but allows for formation of a percolated particulate network that aids in bulk mechanical strength.

Recently, we reported on the synthesis and characterization of novel metal oxide-ionic liquid (IL) ${ }^{24}$ and metal oxide-polyethylene glycol (PEG) hybrid electrolytes based on self-suspended nanoscale organic hybrid materials (NOHMs). ${ }^{25}$ Because they are selfsuspended, these electrolytes are homogeneous fluids, where the tethered units (ILs or PEG oligomers) simultaneously serve as the suspending medium for the nanoparticle cores and as an ion-conducting network for lithium ion transport. These electrolytes have uniformly dispersed nanoparticle cores, unlike the typical composite electrolytes. By manipulating the volume fraction and average size of dispersed particles, it should be possible to design electrolytes that yield and flow under conditions of battery assembly/manufacturing, but which exist as porous ion-conducting "solids" during normal battery operation.

In this article we report on mechanical properties, conductivity, and electrochemical stability of a family of hybrid electrolytes based on concentrated suspensions of $\mathrm{SiO}_{2}$ nanoparticles in oligomeric PEG. In order to create homogeneous suspensions at high silica volume fraction $\phi$, the $\mathrm{SiO}_{2}$ nanoparticles are sterically stabilized by covalently tethering a dense brush of oligo-PEG chains to each particle (Fig. 1B). This approach allows for the creation of suspension electrolytes with a wide range of nanoparticle filler fraction and yet uniformly dispersed, non-percolated, ceramic cores.

\section{Experimental}

\section{Synthesis}

Silica nanoparticles were densely grafted, $\Sigma \approx 1.1$ chains $/ \mathrm{nm}^{2}$, with a polyethylene glycol methyl ether corona (Fig. 2(A)), using our previously reported method, ${ }^{25,26}$ and purified rigorously by repeated precipitation in ethanol. 
Electrolytes were prepared ranging from 0-100 wt $\%$ NOHMs in PEGDME, polyethylene glycol dimethyl ether $\left(M_{\mathrm{n}}=250\right.$, Aldrich) and doped with lithium salt. PEGDME was chosen because it is a low-cost, non-volatile material known to exhibit excellent lithium ion conductivity. A solution of lithium bis(trifluoromethane sulfone imide) (LiTFSI, Aldrich) was prepared in dry methanol (Aldrich) in an Argon-filled glove box (MBraun Labmaster) before use. The lithium salt solution was added to the NOHMs/PEGDME blends at a concentration of $1 \mathrm{M}$ in the organic phase for all samples. After mixing, samples were dried in the convection oven at $50^{\circ} \mathrm{C}$ overnight and for at least 24 hours under high vacuum.

\section{Characterization}

The tethered PEG brush on the purified $\mathrm{SiO}_{2}$ particles was characterized by several methods. Dynamic light scattering (DLS) was performed on a Malvern Instruments Zetasizer Nano-ZS at $25^{\circ} \mathrm{C}$. Intrinsic viscosity of the particles in a low molecular weight polyethylene glycol dimethyl ether host was determined from dilute solution viscosity measurements at $35^{\circ} \mathrm{C}$ using a Rheometrics Scientific ARES rheometer outfitted with $50 \mathrm{~mm}$ parallel plate fixtures. The core particle weight fraction in the hybrids was determined by thermal gravimetric analysis (TGA); heating at $10^{\circ} \mathrm{C} / \mathrm{min}$ to $550^{\circ} \mathrm{C}$.

The dynamic shear rheological properties of the electrolytes was studied using frequency- and straindependent oscillatory shear measurements on an ARES Rheometer and an Anton Paar MCR501. All experiments were performed at $35^{\circ} \mathrm{C}$. Strain sweep measurements were executed at a fixed shear frequency, $\omega=10 \mathrm{rad} / \mathrm{s}$; frequency sweep experiments were performed at a strain within the linear viscoelastic regime for the material $-0.5 \%$ for the stiff hybrid electrolytes and $25 \%$ for the liquid-like materials. Glass transition temperatures of the electrolytes were determined with a TA Differential Scanning Calorimeter at a ramp rate of $10^{\circ} \mathrm{C} / \mathrm{min}$.

\section{Electrochemical measurements}

The ionic conductivity and relaxation frequency of the electrolytes were measured as a function of temperature ( -5 to $100^{\circ} \mathrm{C}$ ) using a Novocontrol Broadband Dielectric Spectrometer. The DC conductivity at each temperature was determined from the plateau value of a plot of $\mathrm{Re}$ [conductivity] as a function of frequency, as described by Joshner, et al. ${ }^{27}$ The segmental relaxation frequency was taken to be the frequency associated with the major peak in $\tan (\delta)$.

Electrochemical stability window, lithium transference number, and interfacial impedance of the hybrid electrolytes were performed on a Solortron Electrochemical Impedance Spectrometer. For all experiments, electrolytes with a high fraction of hybrid was charged to a donut shaped Teflon ring, which prevented short circuiting of the two lithium metal electrodes in the Swagelok stainless steel - Teflon cells. Electrochemical stability windows were determined by linear scan voltammetry at a scan rate of 1 $\mathrm{mV} / \mathrm{s}$, and taken to be the values at the change in slope of the current vs. voltage plot. The lithium transference number of the electrolytes was determined using the method proposed by Bruce ${ }^{28}$ and Scrosati ${ }^{29}$ where initial and steady state values of current are found for a symmetric lithium cell undergoing polarization, with corrections from impedance measurements of the interfacial resistance both before and after polarization.

\section{Results and discussion}

\section{Characterization of nanoscale hybrids}

Dynamic light scattering (DLS) measurements in chloroform (Fig. 2(B)) indicate that the hybrids are comprised of a $2.4 \mathrm{~nm}$ PEG brush tethered to each $\mathrm{SiO}_{2}$ nanoparticle core. Viscosity measurements (Fig. 2(C)) preformed on dilute suspensions of the $\mathrm{SiO}_{2}$-oligo PEG particles in polyethylene glycol dimethyl ether, $M_{\mathrm{n}} \sim 500$, can be fitted using the Einstein formula, $\eta=\mu_{s}(1+[\eta] \phi)$, to yield an intrinsic viscosity $[\eta] \approx 11.1$ for the particles. Here, $\eta$ is the suspension viscosity, $\mu_{s}$ is the viscosity of the suspending medium (PEG-500), and $\phi$ is the volume fraction of $\mathrm{SiO}_{2}$ nanocores in the suspension. The measured intrinsic viscosity is evidently substantially larger than would be expected for a suspension of hard spheres, for which $[\eta]_{\mathrm{HS}}=5 / 2$; the difference can be used to compute the apparent swelling $\Delta \equiv 2 h / d \approx 0.65$ of the particles using the expression, ${ }^{30}[\eta] /[\eta]_{\mathrm{HS}}=(1+\Delta)^{3}$, where the term on the right is a correction to the volume fraction $\phi$ in Einstein's formula to account for the "extra" volume occupied by the tethered polymer chains; $d \approx 7 \mathrm{~nm}$ is the average diameter (Fig. 1(C)) of the un-functionalized $\mathrm{SiO}_{2}$ particles; and $h$ is the hydrodynamic thickness of the PEG brush tethered to the particle. Thus we find $h \approx 2.3 \mathrm{~nm}$, which is satisfyingly close to the value deduced from DLS.

The equilibrium radius of gyration of a PEG chain of average molar mass $M_{w}$ can be computed using the formula, ${ }^{31}$

$$
R_{g}=\frac{1}{\sqrt{6}}\left(C_{\infty} \frac{M_{w}}{m_{0}} l_{m}^{2}\right)^{1 / 2} \approx 6.6 \times 10^{-2} M_{w}^{1 / 2} n m .
$$

Thus for the tethered PEG-trimethoxy silane chains with $M_{w}=660 \mathrm{~g} / \mathrm{mol}, M_{w, P E G} \approx 540 \mathrm{~g} / \mathrm{mol}, R_{g} \approx 1.5 \mathrm{~nm}$ and $h=$ $2 R_{g} \approx 3 \mathrm{~nm}$. Here $\mathrm{C}_{\infty}=5.5$ is the characteristic stiffness ratio for PEG; $l_{m} \approx 0.48 \mathrm{~nm}$ is the size of a statistical segment; and $m_{0}=44$ is the repeat unit molar mass for PEG. A more accurate estimate for the height of the tethered brush, which takes into account crowding and stretching of polymer chains in the brush, can be obtained using de Gennes' blob model for a neutral polymer grafted to a planar substrate modified to account for the spherical geometry of the substrate. This analysis yields $h=\sum_{i} \frac{\xi_{i}}{g_{i}} \approx 3.6 n m$, which is larger than the brush height obtained using both experimental methods. Here $\xi$ is 
the correlation length and $g$ is the number of monomers per correlation volume in each layer of correlation blobs. The fact that the brush height estimated from $R_{g}$ is closer to the values derived from experiment, particularly for the small polymer chain lengths considered here, implies that the PEG chains assume a more collapsed (greater attraction) to the $\mathrm{SiO}_{2}$ substrate than expected for the neutral polymer assumed in the analysis.

\section{Characterization of electrolytes}

The transmission electron microscopy (TEM) image in Fig. 1(B) shows that the PEGDME 250- $\mathrm{SiO}_{2}$ hybrid electrolytes are comprised of homogeneously dispersed nanoparticles in a suspending fluid, with no evidence of aggregation. This latter feature is remarkable for the notoriously "sticky" $\mathrm{SiO}_{2}$ nanocores; it attests to the effectiveness of the grafted PEG oligomers in imparting colloidal stability to $\mathrm{SiO}_{2}$ particles.

Figure 3(A) reports the dynamic shear elastic/storage, $G^{\prime}$, and viscous/loss, $G^{\prime \prime}$, moduli of PEGDME $250-\mathrm{SiO}_{2}$ hybrid electrolytes at various $\mathrm{SiO}_{2}$ volume fractions and shear frequencies, $\omega$. In a typical elastic solid, motion of individual material elements is constrained by interactions with their neighbors and energy storage normal dominates viscous losses, $G^{\prime}>G^{\prime \prime}$. In such a material the elastic moduls is set by interactions between neighboring material elements and generally will not change with shear frequency. At the opposite extreme are hybrid electrolyte suspensions where the viscous, fluid stresses dominate and hence $G^{\prime \prime}>G^{\prime}$, and both are functions of $\phi$. Figure 3(A) shows that for a PEGDME 250- $\mathrm{SiO}_{2}$ hybrid with $\phi=0.19$, the minimum spacing between the particles, $d_{p-p}=\left[\left(\phi_{m} / \phi\right)^{1 / 3}-1\right] d \approx 0.5 d>h$, and $G^{\prime \prime}>>$ $G^{\prime}$, indicative of a fluid-like suspension. At a moderately higher $\mathrm{SiO}_{2}$ volume fraction, $\phi=0.29 ; d_{p-p} \approx 0.3 d \approx h$, the storage modulus increases by more than four orders of magnitude, is greater than the loss modulus over the entire frequency range, and exhibits a much weaker dependence on frequency; indicative of a gel-like solid response. This observation confirms that the tethered PEG chains allow the jammed state to be accessed at lower $\phi$. Figure 3(A) further shows that by $\phi=0.46 ; d_{p-p} \approx 0.1 d<<h, G^{\prime}>>G^{\prime \prime}$ and the dynamic moduli are essentially independent of shear frequency. This means that the suspensions are completely jammed and the tethered polymer brush highly compressed at particle volume fractions well below $\varphi_{m}$.

As pointed out in the introduction, one advantage of a solid-like electrolyte created from a jammed suspension of particles is that, like its thermoplastic polymer host, it is processable. Specifically, at shear stresses above a critical value, $\tau_{y}$, the jammed suspension yields and flows, and as such can be shaped in a typical manufacturing process. Figure 3(B) demonstrates this feature through the effect of shear strain on mechanical moduli. In the small-strain limit $\gamma \rightarrow 0, G^{\prime}$ is independent of strain for all hybrids, which allows us to determine the apparent elastic modulus, $G_{e}=$ $G^{\prime} \lim _{\gamma \rightarrow 0}$, for all materials. At low volume fractions, e.g. $\phi$ $\approx 0.19$, the condition $G^{\prime \prime} \gg G^{\prime}$ is observed at all strains, confirming that the materials are essentially fluids. Additionally, at shear strains order 2, the storage modulus is observed to decrease with increasing strain. Termed strain-softening, ${ }^{30,32}$ this characteristic is commonplace for polymer liquids and is thought to reflect shear alignment of macromolecules in the fluid. ${ }^{32}$

At the next higher volume fraction, $\phi \approx 0.24$, the shear strain at the onset of softening is much lower, in fact more than an order of magnitude lower than for a typical polymer. Additionally, at strains above this value both moduli decrease with increasing strain, and the shear stress (not shown) manifests a noticeable slope change. All of these features are characteristics of yielding, and the shear strain and stress at which the slope change is observed are the yield strain, $\gamma_{y}$, and yield stress $\tau_{y}$ at the conditions of the measurements. At even higher volume fractions, $\phi \geq$ $0.29, \gamma_{y}$ is essentially independent of $\phi$ and the loss modulus, $G^{\prime \prime}$, manifests a pronounced maximum prior to the onset of strain-softening. This maximum is also a characteristic of yielding, ${ }^{33,34}$ it is thought to reflect enhanced viscous dissipation as the cages that constrain motion of individual particles are broken down by the imposed shear. Significantly, back-to-back experiments reveal negligible changes in $\tau_{y}$ and $\gamma_{y}$, and at shear strains beyond the yielding transition, a cross-over to liquid-like flow behaviors, $G^{\prime \prime}>G^{\prime}$, are observed; confirming that moderate shear stress/strain can be used to transform the jammed materials into a processable form, from which it rapidly recovers (becomes jammed again) upon removal of the stress/strain.

Figures 3(C) reports the effect of $\mathrm{SiO}_{2}$ volume fraction on $G_{e}, \tau_{y}$, and $G^{\prime}$. The figure shows that at a volume fraction $\phi=\phi_{y} \approx 0.29$ a dramatic mechanical transformation is observed in which the storage modulus, $G^{\prime}$, increases by over 4 orders of magnitude. These observations reflect a transition from a liquid-like state to a jammed, disordered solid-like material state. A similar transition is seen (Fig. 3(D)) when the low-frequency power-law indices, $G^{\prime} \sim \gamma^{m^{\prime}} ; G^{\prime} \sim \gamma^{m^{\prime \prime}}$, are plotted against $\phi$, also indicative of a transition to a solid-like material at $\phi \approx$ 0.29. In a suspension of hard spheres, the jamming transition is known to occur at $\phi \approx 0.63$. Extrapolation of this result to our hybrid system suggests that the inner 1.1 $\mathrm{nm}$ of tethered oligomers behave mechanically as part of the hard $\mathrm{SiO}_{2}$ nanocore.

Temperature-dependent ionic conductivity for PEGDME $250-\mathrm{SiO}_{2}$ hybrid electrolytes at various $\phi$ is reported in Figure 4(A). The material with $\phi=0.5$ is the selfsuspended/pure $\mathrm{PEG}-\mathrm{SiO}_{2}$ NOHMs, it contains no PEGDME 250. It is immediately evident from the plot that whether they are self- or oligomer-suspended, all hybrid electrolytes studied display Vogel-Thamann-Fulcher (VTF) temperature dependent conductivity, $\mu=A \exp \left(-B /\left(T-T_{0}\right)\right)$, 
over the entire temperature range. Here $B$ is the pseudoactivation energy for coupled ion and local polymer segment motions/breathing modes; $T$ and $T_{0}$ are the measurement and reference temperatures, respectively; and $A$ is a pre-exponential factor. This behavior implies that the materials undergo no melting/crystallization transitions in the temperature range studied; this conclusion is supported by differential scanning calorimetry (DSC) measurements, which are silent in the same temperature range. These observations mean that, consistent with previous studies of $\mathrm{Li}^{+}$diffusion in PEG, segmental motion of the host and tethered PEG chains is important for the conduction mechanism. Table 1 summarizes the pseudoactivation energy $B$ (from the VFT fits) and glass transition temperature $T_{\mathrm{g}}$ (from DSC) for the materials in Figure 4(A). It is noteworthy that the pseudoactivation energy for conduction of the pure NOHMs electrolyte (ie. fraction of tethered chains equal unity) is equivalent to that of the temperature fit of the frequency maximum in the $\tan (\delta)$ (see Supporting Information). This observation is significant because equivalent temperature dependence for these properties confirms that conduction occurs primarily by segmental motion of polymer chains.

Considering the dramatic changes in mechanical properties reported in the last section as hybrids are taken through the jamming transition, one might anticipate as remarkable changes in ionic conductivity. Figures 4(A) and Table 1 show that this is, remarkably, not the case for PEGDME 250- $\mathrm{SiO}_{2}$ hybrid electrolytes. In particular, Figure 4(A) shows that the transition from liquid-like to a jammed physical state produces only a fractional decrease in conductivity at a chosen temperature value. The conductivity decreases noticeably, however, as $\phi$ is increased beyond $\phi_{y}$, and most notably for the pure NOHMs electrolytes. As a group, the hybrid electrolytes manifest the desirable attribute of maintaining both high conductivity and mechanical strength. For example, a material with $\phi=0.38 ; d_{p-p} \approx 0.2 d=1.3 \mathrm{~nm}$ and mean pore

size $a=\frac{2}{3} \frac{(1-\phi)}{\phi} d \approx 1.1 d=7.6 n m$ displays a storage modulus close to $1 \mathrm{MPa}$ and an ionic conductivity of $3.5 \times 10^{-4} \mathrm{~S} / \mathrm{cm}$ at $35^{\circ} \mathrm{C}$, and even a practical conductivity of $1.0 \times 10^{-4} \mathrm{~S} / \mathrm{cm}$ at $10^{\circ} \mathrm{C}$. Likewise, the pseudoactivation energy for conduction in the hybrid with $\phi=0.38$ is around $80 \%$ higher than the value in the hybrid with the lowest $\mathrm{SiO}_{2}$ content $(\phi=0.11)$ ), while the elastic modulus, $G_{\mathrm{e}}$, is close to eight orders of magnitude larger!

That remarkable changes in mechanical properties in the hybrids produce at most modest changes in ionic conductivity suggests that even in a solid-like electrolyte material, ion conduction occurs more or less unimpeded by the jammed structures that produce mechanical reinforcement. The radius of gyration of PEGDME 250 can be estimated using the formula presented earlier, $R_{g} \approx 1 \mathrm{~nm}$. The ionic radius of the bis(trifluoromethanesulfonyl)imide (TFSI) anion has been estimated using hole theory for transport to be $3.62 \AA,{ }^{35}$ substantially larger than the radius of a lithium ion, $0.76 \AA$. The dielectric constant $D$ for PEG of molecular weight 200 has been reported to be 18.43 at $30{ }^{\circ} \mathrm{C},{ }^{36}$ the Debye screening length is $\lambda_{D}=\left(\varepsilon_{0} D R T /\right.$ $\left.2000 F^{2} I\right)^{1 / 2} \approx 1.5 \AA$ at $30{ }^{\circ} \mathrm{C}$ for a $1 \mathrm{M}$ solution of LiTFSI in PEG. Here $F$ is the faraday charge; $\varepsilon_{0}$ is the vacuum permittivity; and $I$ is the ionic strength of the electrolyte. Thus for the PEGDME 250-SiO 2 hybrid electrolyte with $\phi$ $=0.38$ discussed above, PEG chains are strongly confined and TFSI ions can move, at best in single-file, undisturbed by the smallest bottlenecks in the porous material. For the pure NOHMs electrolyte, $\phi=0.55 ; d_{p-p} \approx 0.05 d=3.2 \AA$, even single-file passage of TFSI through the bottleneck region is on average disallowed. However, the mean pore diameter, $a \approx 0.55 d=3.8 \mathrm{~nm}$, in the "continuum" representation of the material is still large enough to allow bulk-like ion transport in the pores.

If ion transport in PEGDME 250- $\mathrm{SiO}_{2}$ hybrid electrolytes occur by the same mechanisms as in bulk PEG, the ionic conductivity should be to leading order proportional to the "void fraction", $(1-\phi)$. This statement is explored in Figure 4(B) where the conductivity is plotted against void fraction at various temperature distances from the glass transition, $T_{r}=T-T_{g}$. Surprisingly, the figure shows that with the possible exception of the pure NOHMs electrolyte, the ionic conductivity is in fact proportional to the void fraction over the entire range of particle volume fractions. The figure also shows that the proportionality constant increases with increasing $T_{r}$, and that the jamming transition has no effect on ionic conductivity. Thermal and ionic conductivity in particle suspensions have been studied by several groups, ${ }^{37-39}$ using a framework proposed by Maxwell. ${ }^{40}$ In this model, the effective conductivity, $\sigma$, of a homogeneous suspension of particles of conductivity, $\sigma_{p}$, volume fraction, $\varphi$, dispersed in a medium of conductivity, $\sigma_{0}$, can be computed using a simple formula, $\sigma / \sigma_{0}=(1-$ $2 \alpha \phi) /(1+\alpha \phi)$, where $\alpha=\left(\sigma_{0}-\sigma_{p}\right) /\left(2 \sigma_{0}+\sigma_{p}\right)$ and $\sigma / \sigma_{0}$ is the relative conductance at the specified temperature. In the limiting case where the particles are perfect insulators $\alpha=$ $1 / 2$ and Maxwell's formula becomes, $\sigma / \sigma_{0}=2(1-\phi) /(2+$ $\phi)$.

The inset in Figure 4(B) shows several important features of the ionic conductivity in our hybrid electrolytes. First, the strong temperature dependence seen in the main figure essentially disappears when the conductivity is expressed in terms of the relative conductivity at the same $T_{\mathrm{r}}$. This means that the conduction mechanism in the hybrids is the same as in free PEG. Additionally, it means that the change in shape of the conductivity versus temperature plots in Figure 4(A) originates from the effect of $\phi$ on $T_{\mathrm{g}}$. Second, the figure shows that when plotted in the Maxwell form, assuming that $\mathrm{SiO}_{2}$ is an ionic insulator, the relative conductivity is a linear function of the abscissa variable for all values of $\phi$, including the pure NOHMs electrolyte. This result is remarkable because it means that the simple physics in the Maxwell expression are applicable over the entire range of volume fraction of $\mathrm{SiO}_{2}$ in the hybrids. The uniform particle spacing produced by 
the tethered PEG chains allows for this favorable result, which varies from the properties expected of a system with a percolated non-conducting phase where conduction is markedly inhibited. It is important to point out, however, that the slope of the line in the inset is 3.5 , compared to the theoretical result of 2 . This difference implies that the $\mathrm{SiO}_{2}$ particles do make a non-negligible contribution to the ionic conductivity of the hybrids, likely because the tethered PEG chains are able to interact with $\mathrm{Li}^{+}$which reduces its mobility. Based on findings from our previous study which showed that change in hybrid core chemistry from $\mathrm{SiO}_{2}$ to $\mathrm{TiO}_{2}$ had no effect on the conduction properties, ${ }^{25}$ we believe that the dense functionalization of the nanoparticle surface with oligomers inhibits the anion adsorption effect seen in "soggy-sand" electrolytes.

Electrochemical stability measurements were conducted on a jammed suspension electrolyte, $\phi=0.3$, in a symmetric, lithium/electrolyte/lithium, configuration in Swagelok cells. Figure 5(A) shows the impedance response at various intervals in time; the points are the data and lines are fits to the equivalent circuit model in the inset. This circuit model has been used previously to explain the impedance response of electrolytes in symmetric lithium cells, ${ }^{42}$ where $R_{\mathrm{b}}$ is the bulk electrolyte resistance, $R_{\text {int1 }}$ and $R_{\text {int2 }}$ are interfacial resistances, $\mathrm{Q}_{1}$ and $\mathrm{Q}_{2}$ are the corresponding constant phase element capacitances, and $\mathrm{W}$ is the Warburg diffusion element. Figure 5(B) summarizes the magnitude of the bulk and interfacial resistances, which near steady-state values over a 1000-hour evaluation period. Consistent interfacial resistances and impedance response means that a stable solid electrolyte interfacial (SEI) layer is formed with metallic lithium. Figure $5(\mathrm{C})$ shows the electrochemical stability window for both the jammed hybrid and pure PEGDME electrolytes in contact with lithium. Like most PEG/PEO based electrolytes, the cathodic stability is about $0.3 \mathrm{~V}$; the anodic stability of this electrolyte is around $6.3 \mathrm{~V}$. This wide electrochemical stability window allows for the potential use of this electrolyte with a range of electrode materials. Finally, the lithium transference number, the fraction of the ionic conductivity due to lithium transport, was determined by the Bruce/Scrosati method: ${ }^{28,29}$ $T_{L i^{+}}=\frac{I_{s s}\left(\Delta V-I_{0} R_{0}\right)}{I_{0}\left(\Delta V-I_{s s} R_{s s}\right)}$ where $I_{0}$ and $I_{\mathrm{ss}}$ are the initial and steady-state current of a cell undergoing a polarization potential of $\Delta \mathrm{V}$, and $R_{0}$ and $R_{\mathrm{ss}}$ and the interface charge transfer resistances before and after polarization. The transference number was determined to be less than unity, as expected for a PEG based system: $T_{\mathrm{Li}^{+}}=0.4 \pm 0.1$ when $I_{0}$ was taken to be the actual measured initial current and $T_{\mathrm{Li}+}=0.65 \pm 0.1$ when $I_{0}$ is taken to be the initial current as estimated by fitting the transient current to a single exponential decay (see Supporting Information).

\section{Conclusions}

We have shown in this work that mechanically tunable, processable, hybrid electrolytes can be created using jammed, homogeneous suspensions of $\mathrm{SiO}_{2}$-PEG hybrid particles dispersed in oligomeric PEG hosts. Notably, the jamming transition that yields marked - desirable increases in mechanical moduli produces only modest changes in ionic conductivity. The effective medium model of Maxwell is used to show that even at $\mathrm{SiO}_{2}$ core fractions where the inter-particle "throat" diameters in the jammed hybrids are close to the anion size, the conduction processes are largely unaffected by the porous, tortuous network of channels created by the $\mathrm{SiO}_{2}$ network. Significantly, we also find that these hybrid electrolytes manifest reasonable lithium transference numbers and good electrochemical and interface stability in the presence of lithium. Together, these features make them promising candidates for application in advanced secondary lithium metal batteries; wherein the highly tortuous, nanometersized fluid pathways between the jammed $\mathrm{SiO}_{2}$ particles are expected to frustrate/arrest lithium dendrite growth and proliferation after repeated charge/discharge cycles.

\section{Acknowledgements}

This work was supported by Award No. KUS-C1-018-02, made by King Abdullah University of Science and Technology (KAUST), and by the National Science Foundation, Award No. DMR-1006323. JLN also acknowledges support from the Materials for a Sustainable Future IGERT program, NSF grant \# DGE-0903653.

\section{Notes and references}

address: School of Chemical and Biomolecular Engineering, Cornell University, Ithaca, New York, $14853 *$ Fax: 607-255-9166 Tel:607-254-8825E-mail: laa25@cornell.edu

$\dagger$ Electronic Supplementary Information (ESI) available: [synthesis scheme, TGA data, power law frequency dependence of the moduli, $\tan (\delta)$ VFT fit, lithium transference number data]. See DOI: $10.1039 / \mathrm{b} 000000 \mathrm{x} /$

1. F. Wang, J. Yang, Y. NuLi, and J. Wang, $J$. Power Sources, 2010, 195, 6884.

2. J.-i.Yamaki, S.-i. Tobishima, K. Hayashi, K. Saito, Y. Nemoto and M. Arakawa, J. of Power Sources, 1998, 74, 219.

3. R. Bhattacharyya, B. Key, H. Chen, A. S. Best, A. F. Hollenkamp and C. P. Grey, Nature Mater. 2010, 9, 504.

4. B. Scrosati and C. Vincent, Mater. Res. Bull. 2000, 28 .

5. P. G. Bruce, B. Scrosati and J.-M. Tarascon, Angew. Chem., Int. Ed. 2008, 47, 2930. 
6. Y.-J. Choi, Y.-D. Chung, C.-Y. Baek, and K.-W. Kim, J. Power Sources, 2008, 184, 548.

7. M. B. Armand, J. M. Chabagno and M. J. Duclot, Fast Ion Transport in Solids. ed.; NorthHolland: New York, 1979; p 131.

8. S. Sylla, J.-Y. Sanchez and M. Armand, Electrochim. Acta, 1992, 37, 1699.

9. P. Villano, M. Carewska, G. B. Appetecchi and S. Passerini, J. Electrochem. Soc., 2002, 149, A1282.

10. M. Marzantowicz, J. R. Dygas, F. Krok, A. Tomaszewska, Z. Florjanczyk, E. ZygadloMonikowska and G. Lapienis, J. of Power Sources, 2009, 194, 51.

11. M. Singh, O. Odusanya, G. M. Wilmes, H. B. Eitouni, E. D. Gomez, A. J. Patel, V. L. Chen, M. J. Park, P. Fragouli, H. Iatrou, N. Hadjichristidis, D. Cookson, and N. Balsara, Macromolecules, 2007, 40, 4578.

12. F. Croce, G. B. Appetecchi, L. Persi and B. Scrosati, Nature, 1998, 394, 456; F. Croce, R. Curini, A. Martinelli, L. Persi, F. Ronci, B. Scrosati and R. Caminiti, J. Phys. Chem. B, 1999, 103, 10632.

13. C. Capiglia, P. Mustarelli, E. Quartarone, C. Tomasi and A. Magistris, Solid State Ionics, 1999, 118, 73.

14. M. Reddy, P. Chu, J. Kumar and U. Rao, J. Power Sources, 2006, 161, 535.

15. H. J. Walls, M. W. Riley, R. R. Singhal, R. J. Spontak, P. S. Fedkiw, and S.A. Khan, $A d v$. Funct. Mater., 2003, 13, 710.

16. H.-M. Kao and C.-L. Chen, Angew. Chem., Int. Ed., 2004, 43, 980.

17. H.-M. Xiong, D.-P. Liu, H. Zhang and J.-S. Chen, J. Mater. Chem., 2004, 14, 2775.

18. P. Maitra and S. Wunder, Chem. Mater ., 2002, 14, 4494.

19. J.-M. Tarascon, A. S. Gozdz, C. Schmutz, F. Shokoohi and P. C. Warren, Solid State Ionics, 1996, 86-88, 49.

20. H. Ye, Huang, J. J. Xu, A. Khalfan and S. G. Greenbaum, S. G. J. Electrochem. Soc., 2007, 154, A1048.

21. S. Seki, Y. Ohno, Y. Kobayashi, H. Miyashiro, A. Usami, Y. Mita, H. Tokuda, M. Watanabe, K.
Hayamizu, S. Tsuzuki, M. Hattori and N. Terada, J. Electrochem. Soc. 2007, 154, A173.

22. P. Birke, F. Salam, S. Doring and W. Weppner, Solid State Ionics, 1999, 118, 149.

23. D. K. Pradhan, R. N. P. Choudhary and B. K. Samantaray, Mater. Chem. Phys., 2009, 115, 557.

24. S. S. Moganty, N. Jayaprakash, J. L. Nugent, J. Shen, and L. A. Archer, Angew. Chem. Inter. Ed., 2010, 49, 9158.

25. J. L. Nugent, S. S. Moganty and L. A. Archer, Adv. Mater. 2010, 22, 3677.

26. Q. Zhang and L. A. Archer, Langmuir, 2002, 18, 10435 .

27. A. K. Jonscher, Nature (London). 1977, 267, 673.

28. P. G. Bruce, J. Evans and C. A. Vincent, Solid State Ion., 1988, 28-30, 918.

29. G. B. Appetecchi, G. Dautzenberg and B. Scrosati, J. Electrochem. Soc., 1996, 143, 6.

30. R. G. Larson, The Structure and Rheology of Complex Fluids, 1999, Oxford, New York.

31. M. Rubinstein and R. Colby, Polymer Physics, 2004, Oxford, New York.

32. M. Doi and S. F. Edwards, The Theory of Polymer Dynamics 1980, Cambridge University Press, New York.

33. W. J. Frith, T. A. Strivens and J. Mewis, J. Colloid and Interface Sci., 1990, 139, 55.

34. P. Agarwal, H. Qi and L. A. Archer, Nano Lett., 2010, 10, 111.

35. A. T. Abbott, Chem. Phys. Chem., 2004, 5, 1242.

36. A. M. Awwad, H. Al-Dujaili and H. E. Salman, J. Chem. Eng. Data, 2002, 47, 421.

37. W. Yu and S. U. S. Choi, Journal of Nanoparticle Research, 2003, 5, 167.

38. M. Pavlin, T. Slivnik and D. Miklavčič, D. IEEE Tran. Biomed. Eng., 2000, 49, 77.

39. H. Cheng and S. Torquoto, Proc. Royal Soc. Lond. A, 1997, 453, 145. 
40. J.C. Maxwell, Treatise on Electricity and Magnetism, 1873, Oxford University Press, London, UK.

41. A. J. Bhattacharyya, M. Dolle, and J. Maier, Electrochem. and Solid-State Lett., 2004, 7, A432.

42. S. Liu, N. Imanishi, T. Zhang, A. Hirano, Y Takeda, O. Yamamoto and J. Yang, Journal of Power Sources, 2010, 195, 6847. 


\section{Figure Captions}

Figure 1. Hydraulically interconnected framework: (a) Schematic - grey circles represent impermeable particles; green and brown dots represent the lithium cations and TFSI anions, respectively. (b) TEM micrograph of a PEGDME 250- $\mathrm{SiO}_{2}$ hybrid electolyte with $\phi \approx 0.3$.

Figure 2. $\mathrm{SiO}_{2}$-PEG NOHMs hybrids: (a) Schematic of polymer chain bonded to silica core with associated ions. (b) Dynamic light scattering measurements of the base silica and purified hybrid. (c) Plot of relative viscosity vs. $\phi$ used to determine the intrinsic viscosity of the hybrid units.

Figure 3. Rheology: (a) Storage modulus, $G^{\prime}$, (filled symbols) and loss modulus, $G^{\prime \prime}$,(open symbols) of PEGDME 250- $\mathrm{SiO}_{2}$ hybrid electrolytes with varying $\varphi$, as a function of shear frequency, (b) $G^{\prime}$ and $G^{\prime \prime}$ of the electrolytes as a function of strain, $\gamma$. (c) $G^{\prime}$ as obtained from frequency sweep measurements in the linear viscoelastic regime; $G_{e}$ and $\tau_{y}$ as obtained from strain sweep measurements at $\omega=10 \mathrm{rad} / \mathrm{s}$ as a function of $\phi$. (d) Slopes $m$ ' and $m$ " of the power law dependance of $G^{\prime}$ and $G^{\prime}$ from frequency sweep measurements.

Figure 4. Ionic Conductivity: (a) Full range of electrolytes from pure plasticizer to pure hybrid. Points are the actual data and lines are the VTF fits. (b) Temperature reduced conductivity $\sigma_{\mathrm{Tr}}$, where $T_{r}=T-T_{g}$ for $T_{r}=75,90$, $120,150,180 \mathrm{~K}$.

Figure 5. Electrochemical Characterization: (a) Interfacial charge transfer resistance of an aging cell at discrete points in time. Lines are fits to the displayed equivalent circuit model. (b) Values of the resistances $R_{\text {int } 1}$, $R_{\text {int } 2}$, and $R_{\mathrm{b}}$, determined by circuit fitting, as a function of time. (c) Electrochemical stability windows of pure PEGDME electrolyte $(\phi=0)$ in red, and plasticized NOHMs electrolyte $(\phi=0.3)$ in black.

Table 1: Pseudo activation energy, $B$, of ionic conduction and glass transition temperature, $T_{g}$, of electrolytes as a function of $\mathrm{SiO}_{2}$ volume fraction, $\phi$. 


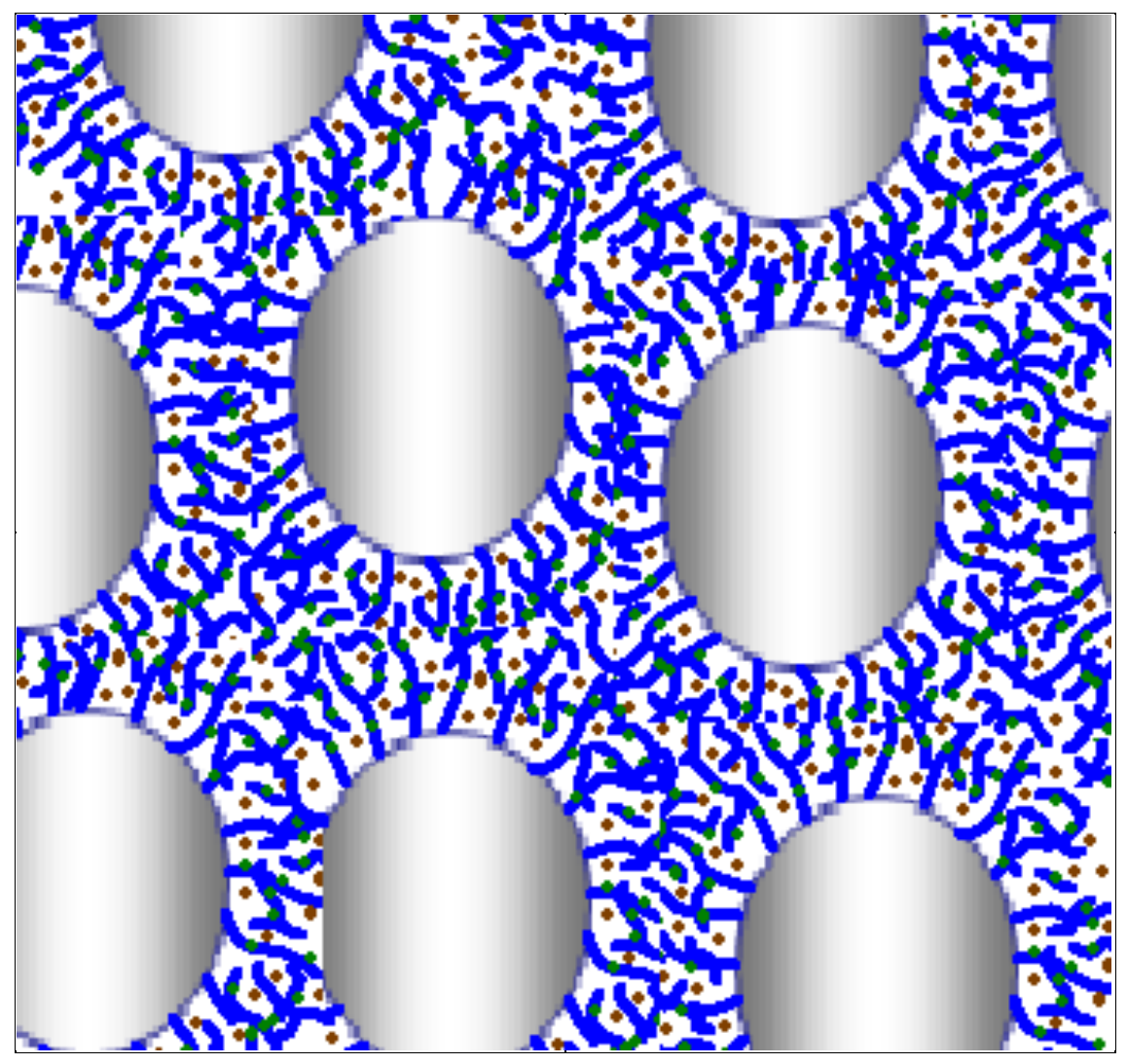

Figure 1(A) 


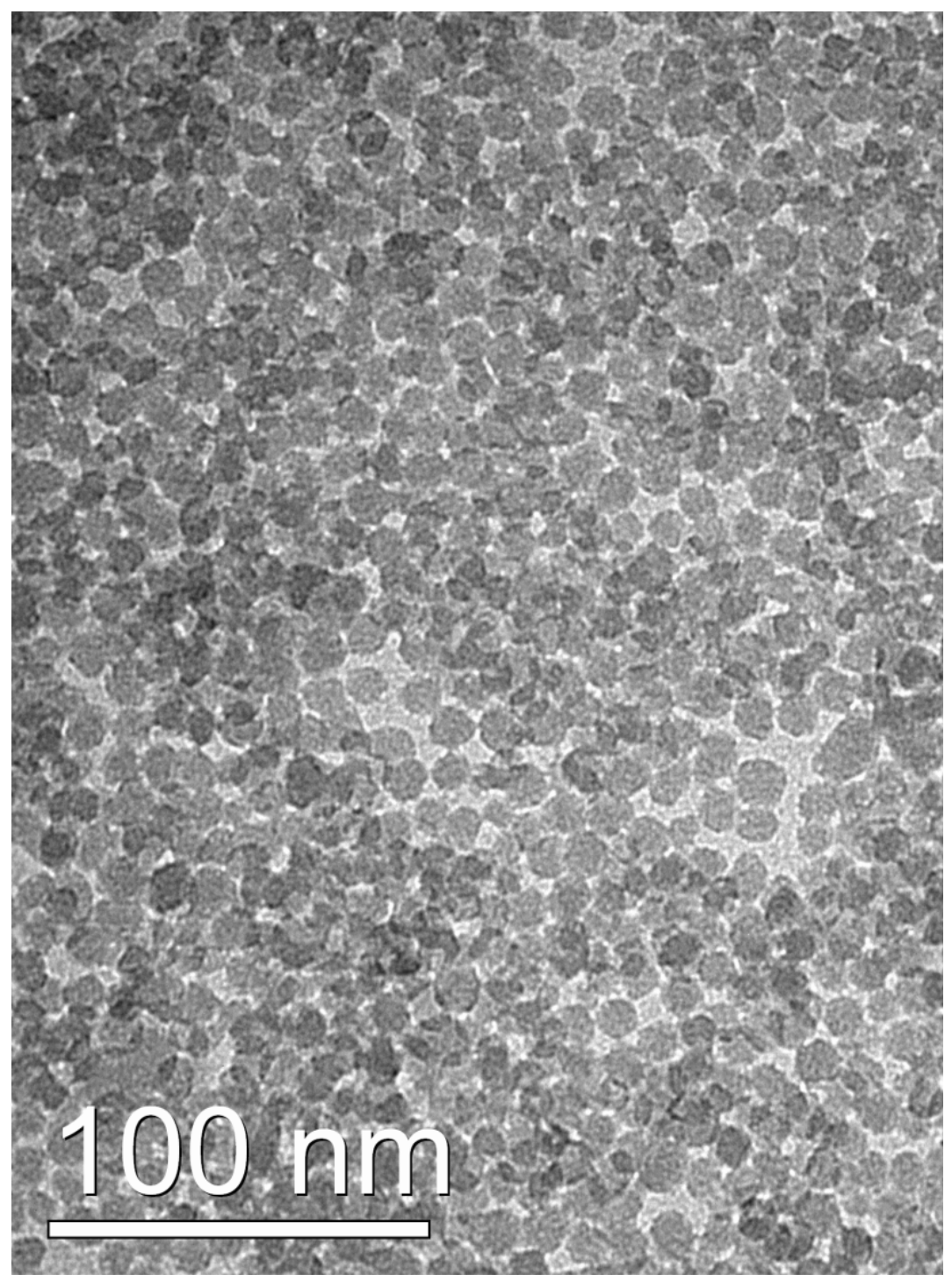

Figure 1(B) 


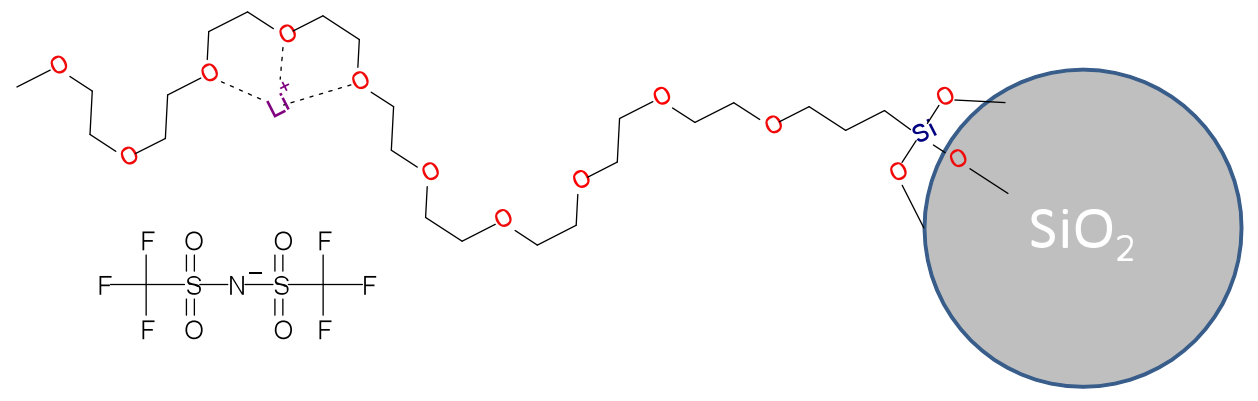

Figure 2(A) 


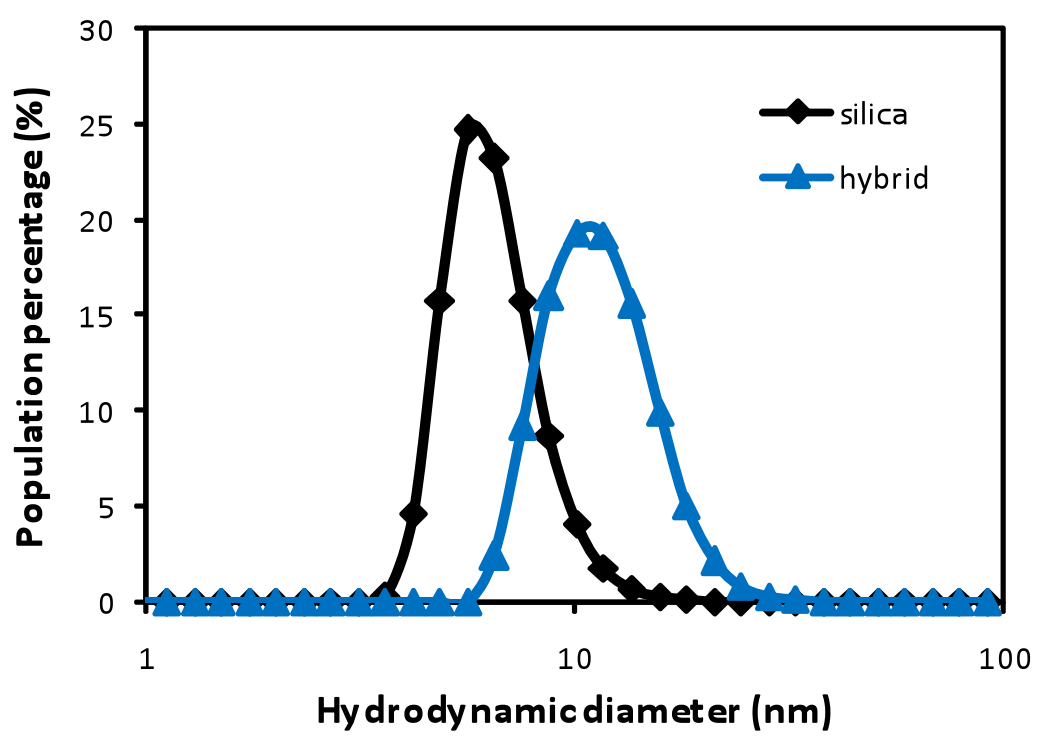

Figure 2(B) 


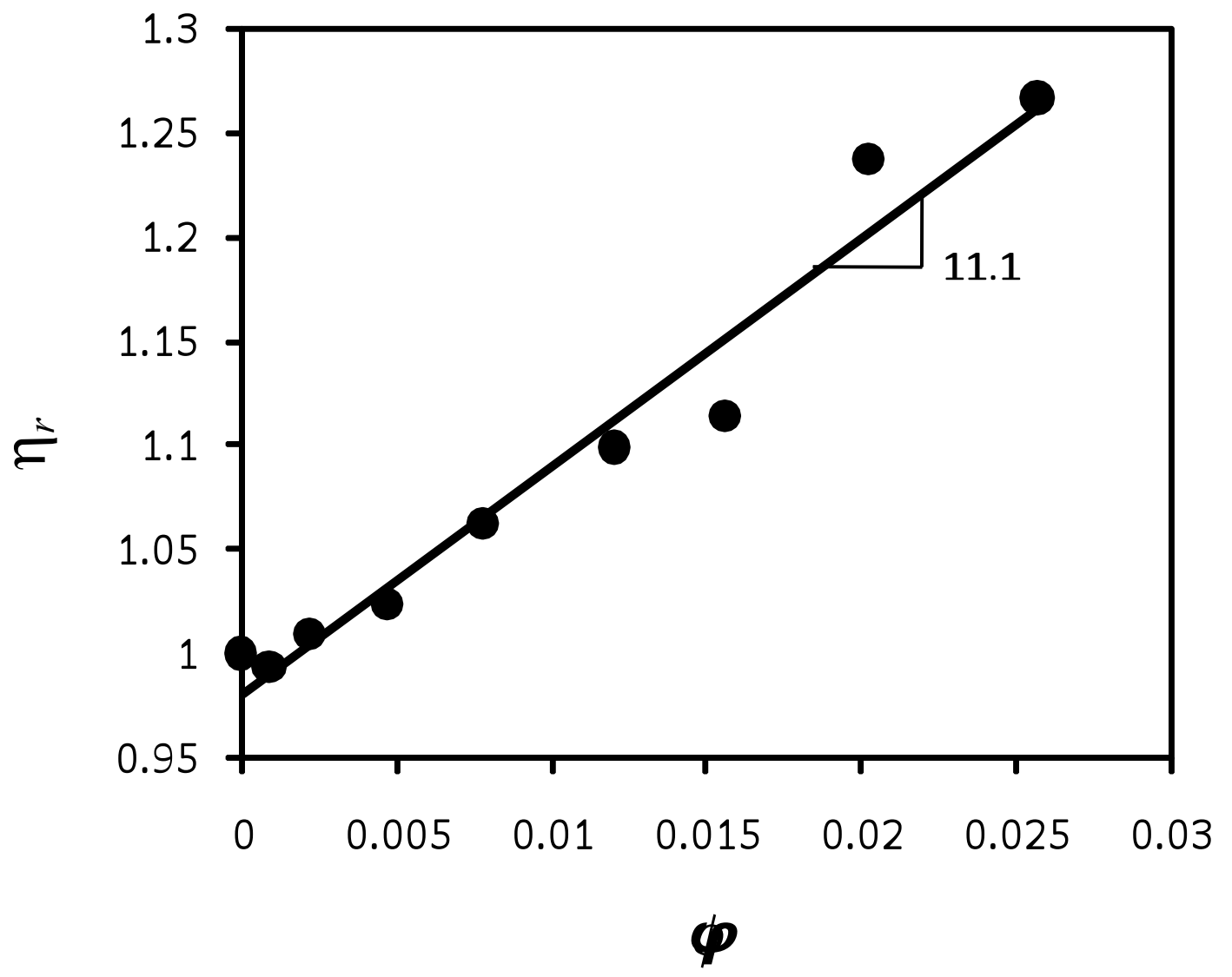

Figure 2(C) 


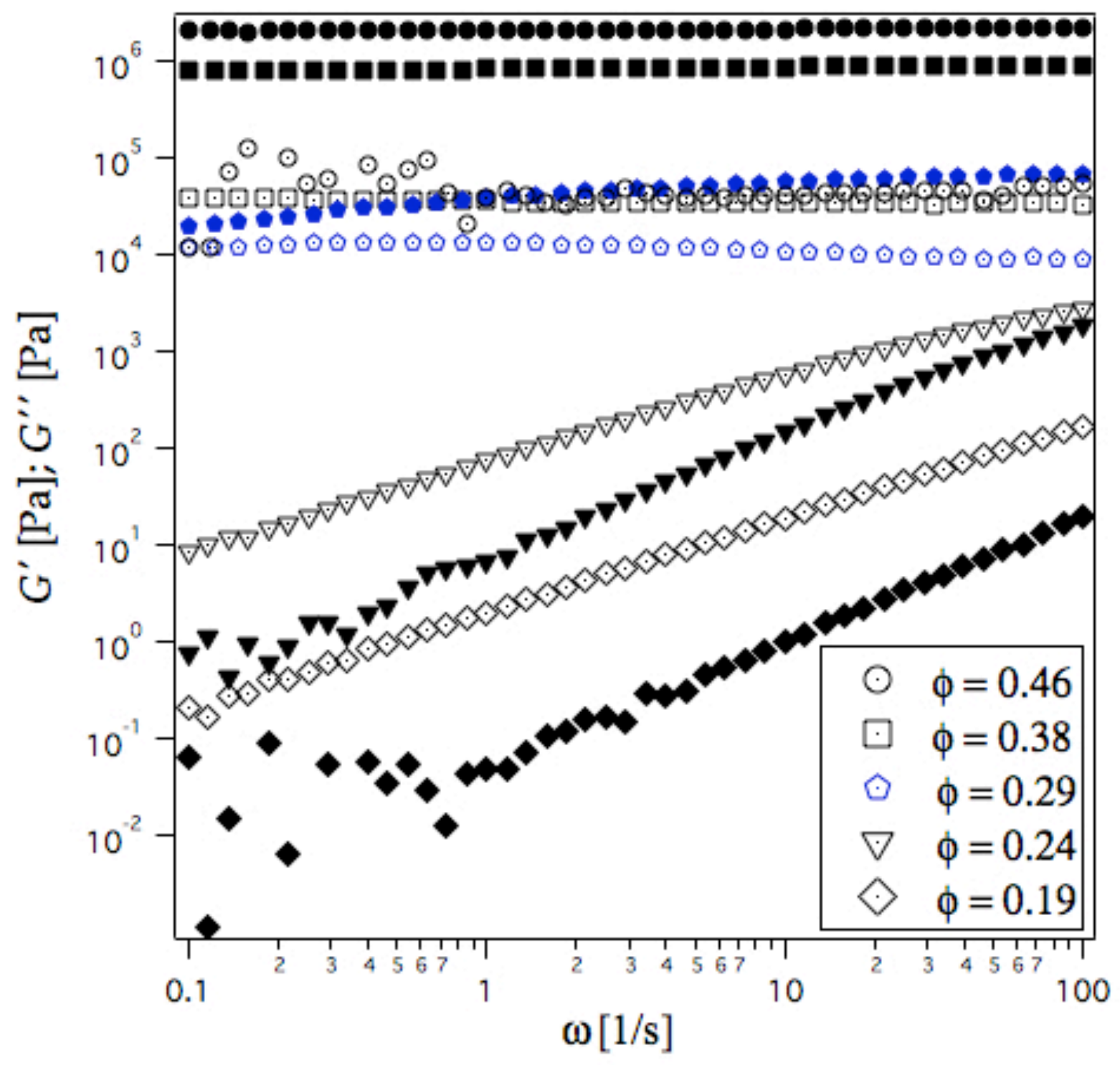

Figure 3(A) 


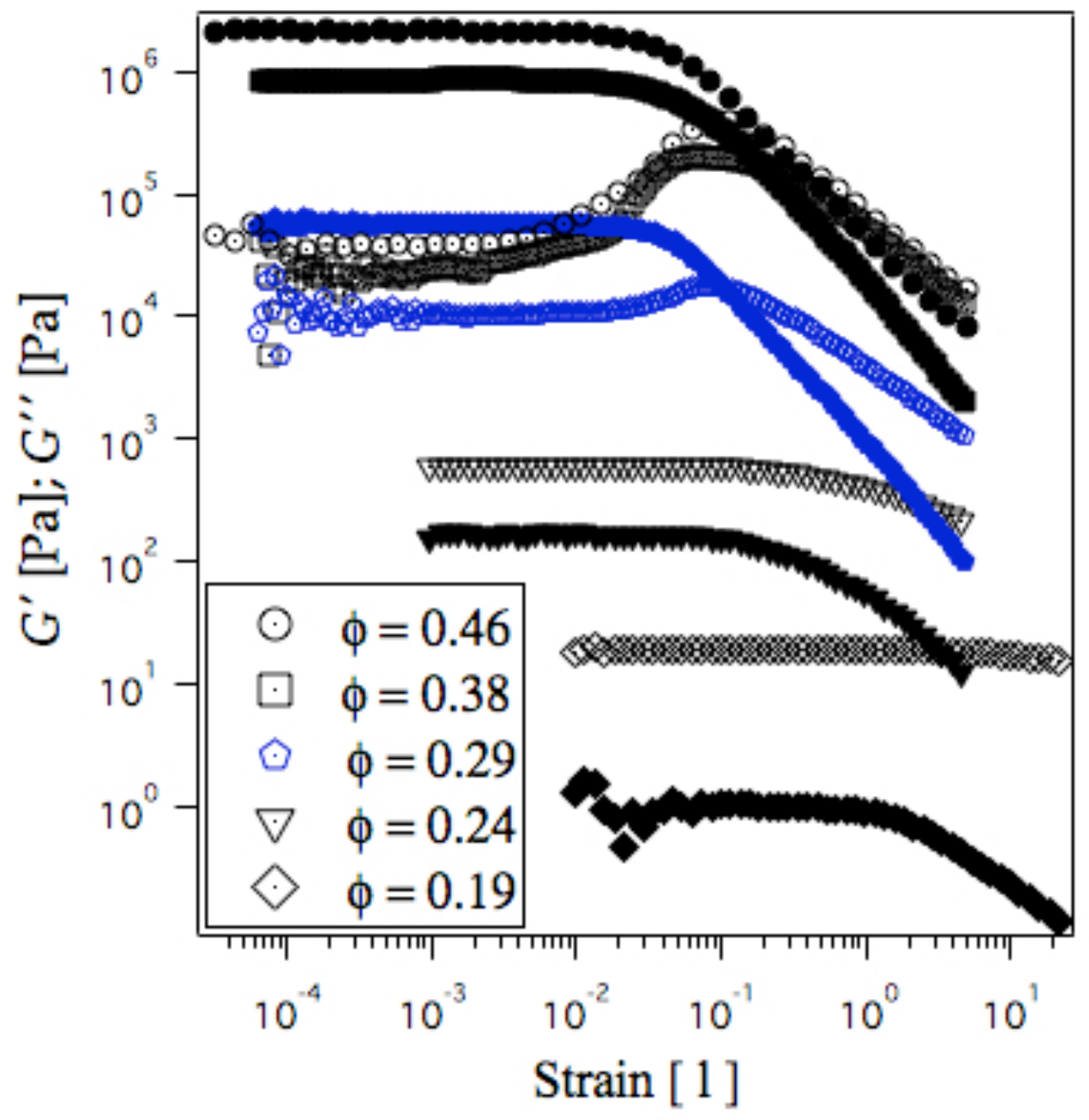

Figure 3(B) 


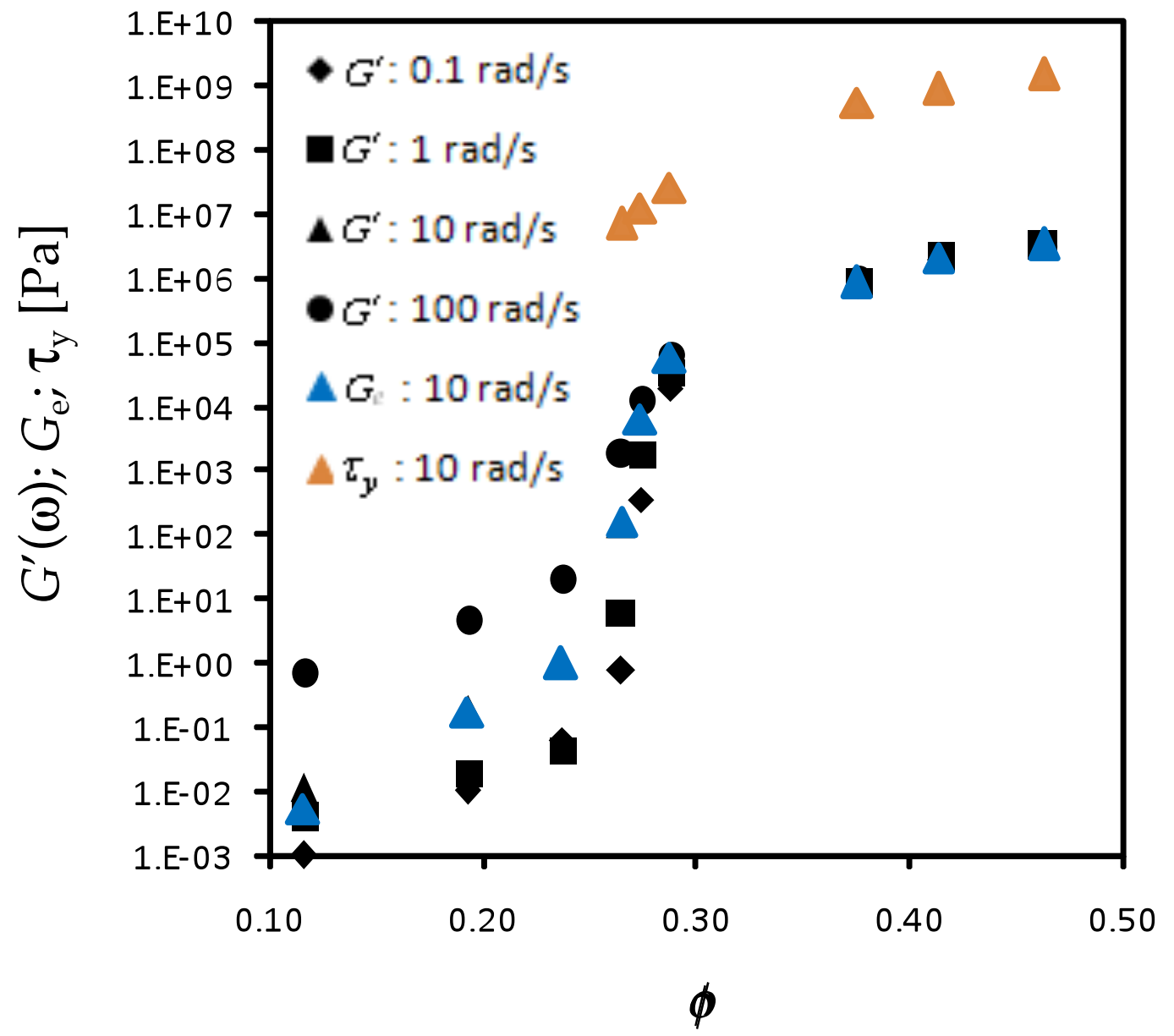

Figure 3(C) 


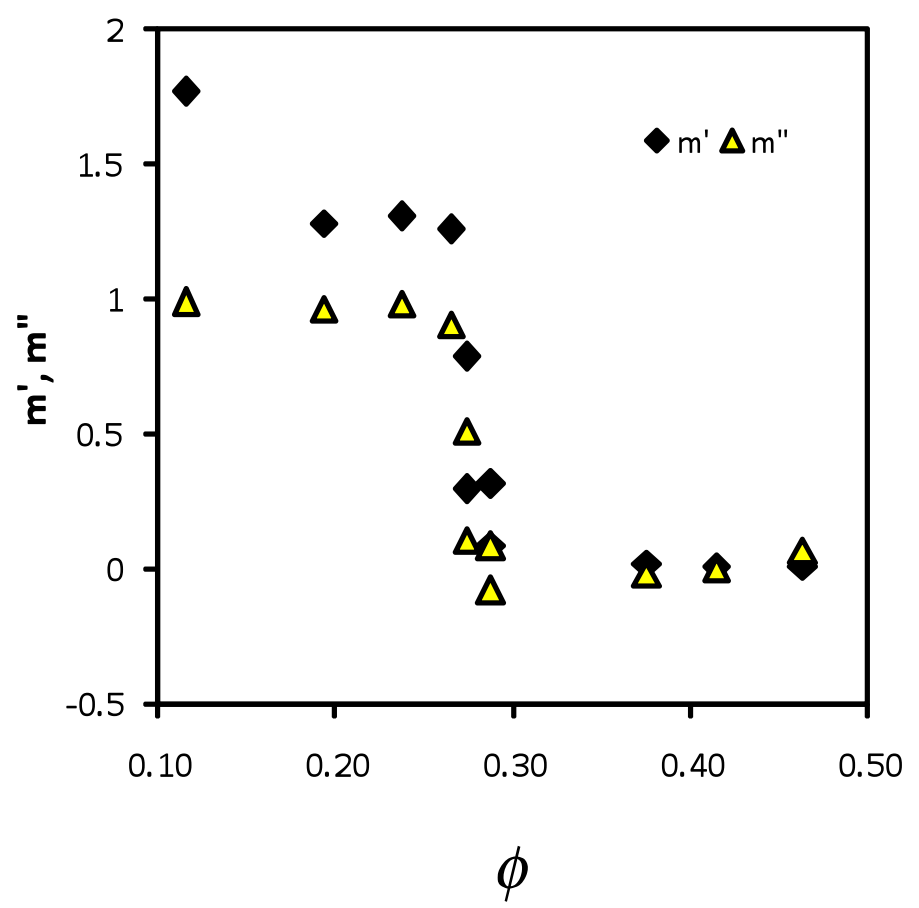

Figure 3(D) 


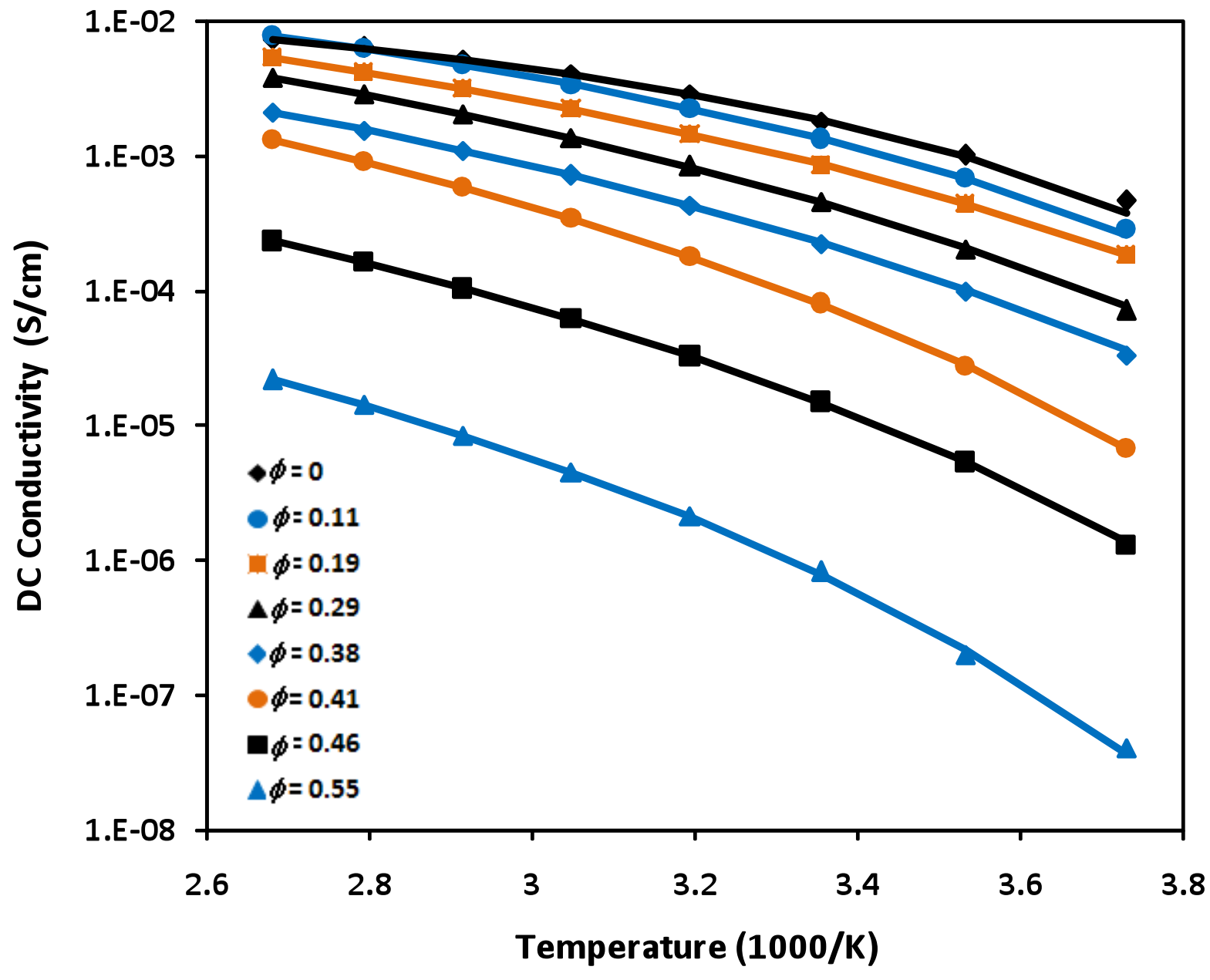

Figure 4(A) 


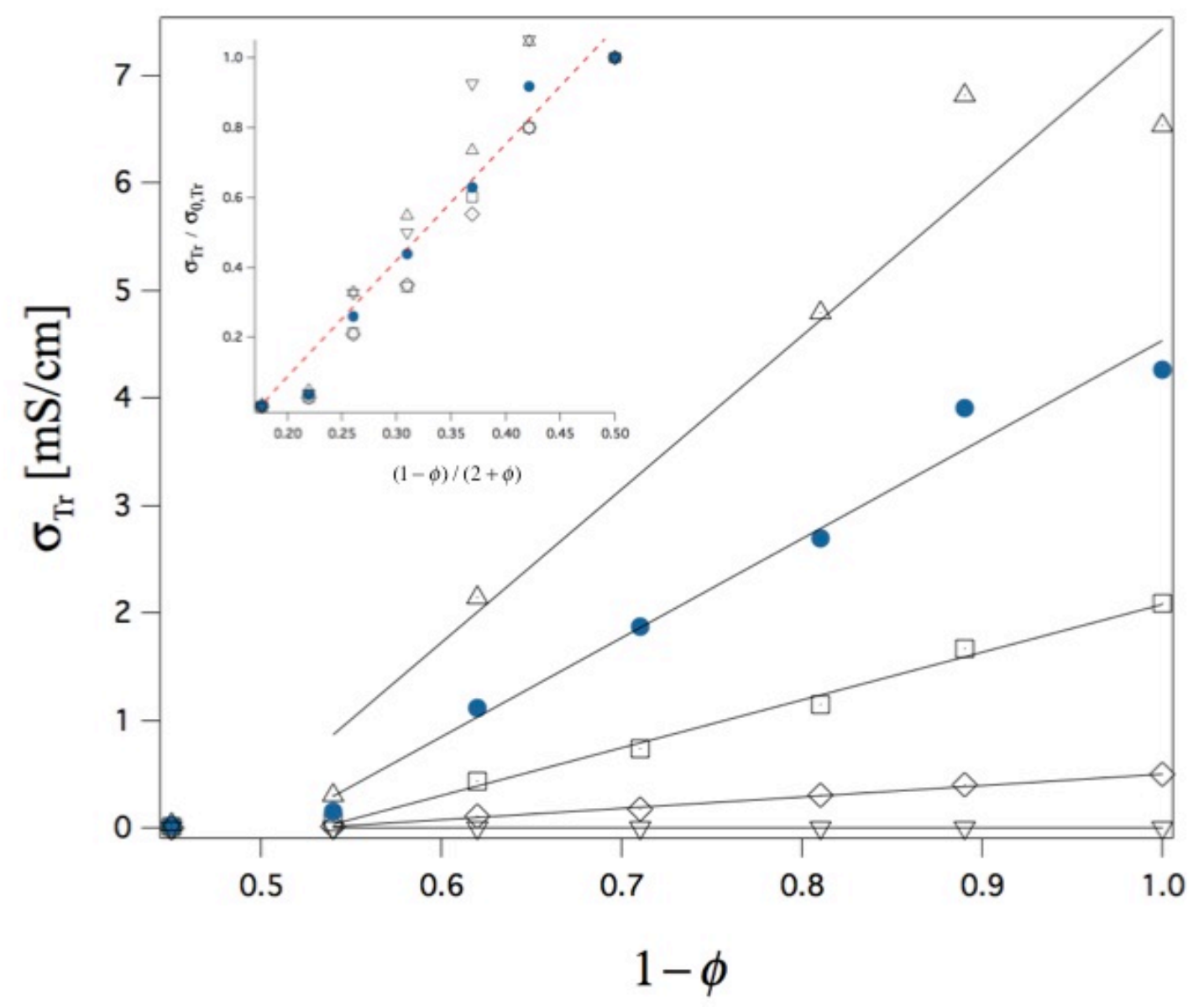

Figure 4(B) 


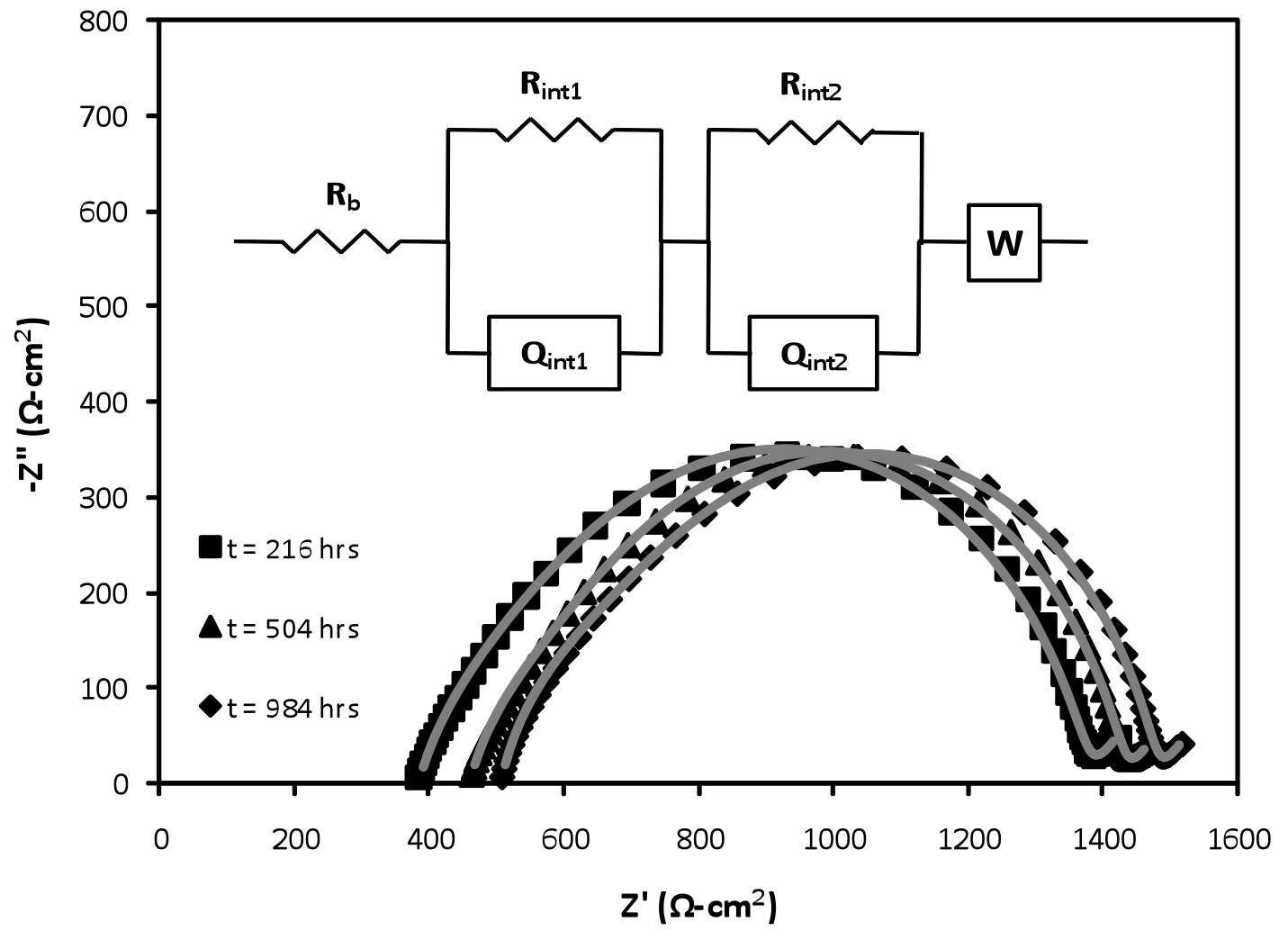

Figure 5(A) 


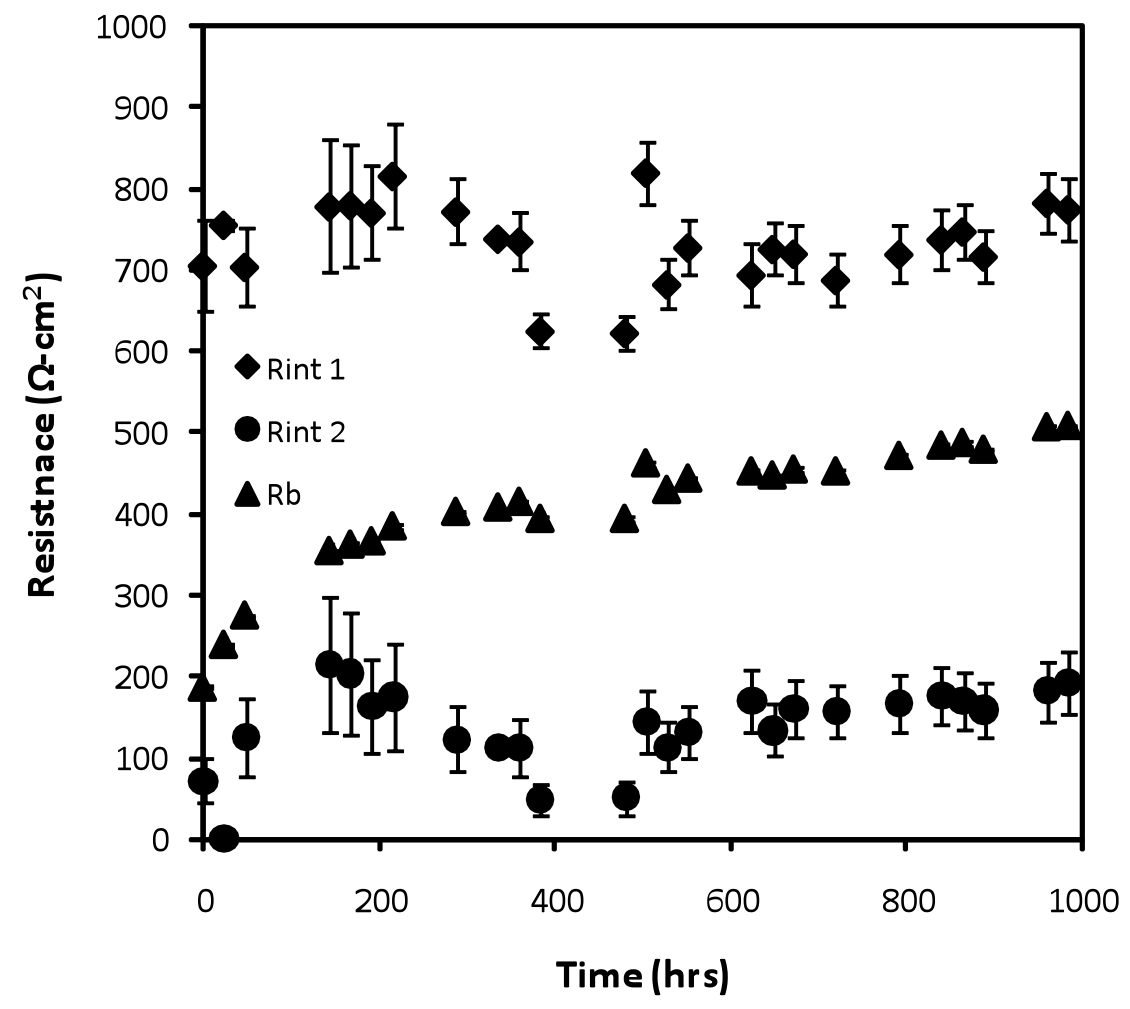

Figure 5(B) 


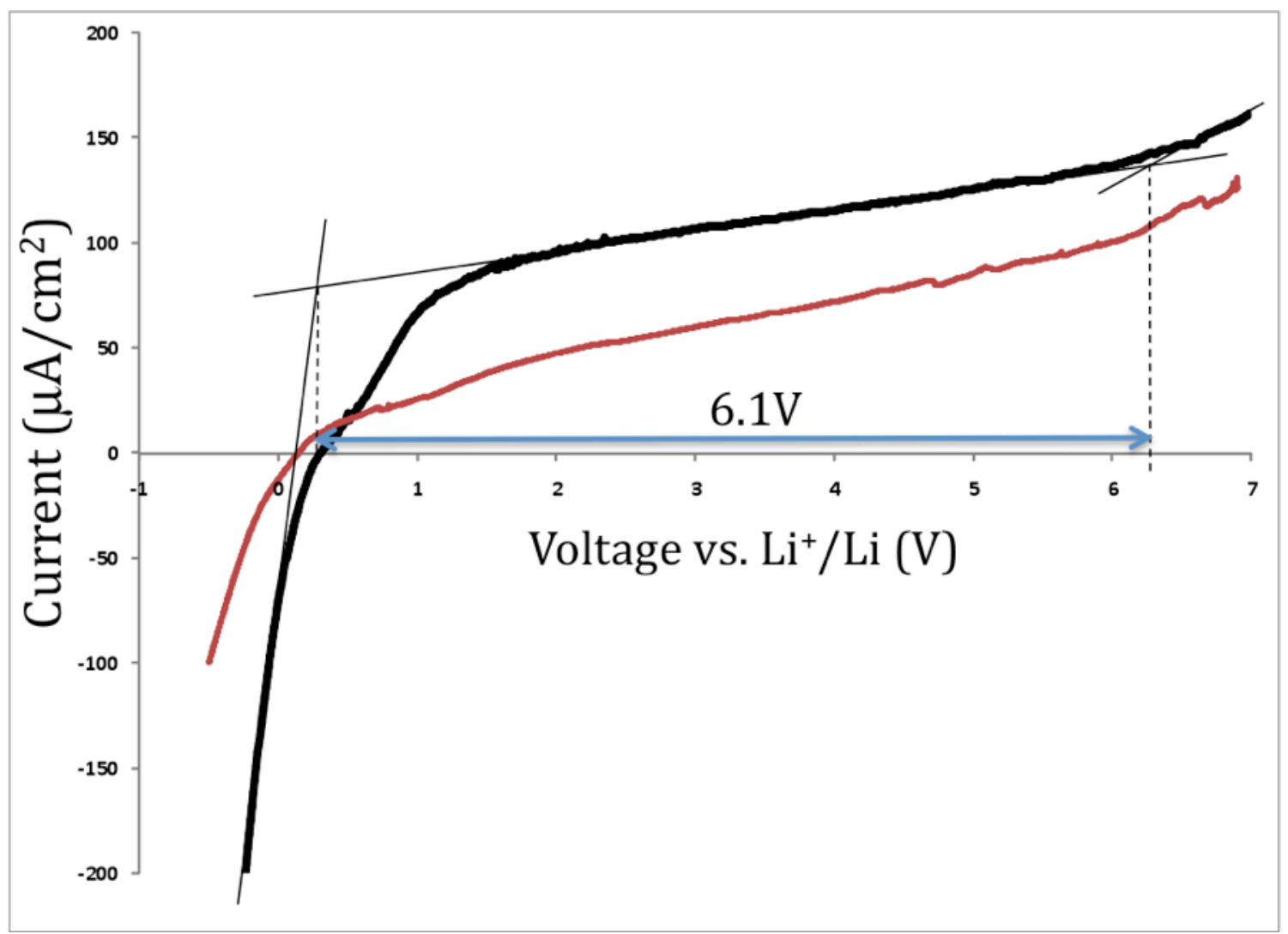

Figure 5(C) 
Table 1.

\begin{tabular}{|c|c|c|}
\hline$\phi$ & $B[K]$ & $\boldsymbol{T}_{g}[\mathrm{~K}]$ \\
\hline 0 & $252 \pm 26$ & 181.5 \\
\hline 0.11 & $475 \pm 30$ & 184 \\
\hline 0.19 & $592 \pm 5$ & 186 \\
\hline 0.29 & $718 \pm 17$ & 189.5 \\
\hline 0.38 & $790 \pm 38$ & 194 \\
\hline 0.46 & $856 \pm 14$ & 206 \\
\hline 0.55 & $904 \pm 84$ & 213 \\
\hline
\end{tabular}

